Rev. Mat. Iberoamericana 23 (2007), no. 2, 513-536

\title{
On a Parabolic Symmetry Problem
}

\author{
John L. Lewis and Kaj Nyström
}

\begin{abstract}
In this paper we prove a symmetry theorem for the Green function associated to the heat equation in a certain class of bounded domains $\Omega \subset \mathbb{R}^{n+1}$. For $T>0$, let $\Omega_{T}=\Omega \cap\left[\mathbb{R}^{n} \times(0, T)\right]$ and let $G$ be the Green function of $\Omega_{T}$ with pole at $(0,0) \in \partial_{p} \Omega_{T}$. Assume that the adjoint caloric measure in $\Omega_{T}$ defined with respect to $(0,0), \hat{\omega}$, is absolutely continuous with respect to a certain surface measure, $\sigma$, on $\partial_{p} \Omega_{T}$. Our main result states that if

$$
\frac{d \hat{\omega}}{d \sigma}(X, t)=\lambda \frac{|X|}{2 t}
$$

for all $(X, t) \in \partial_{p} \Omega_{T} \backslash\{(X, t): t=0\}$ and for some $\lambda>0$, then $\partial_{p} \Omega_{T} \subseteq\{(X, t): W(X, t)=\lambda\}$ where $W(X, t)$ is the heat kernel and $G=W-\lambda$ in $\Omega_{T}$. This result has previously been proven by Lewis and Vogel under stronger assumptions on $\Omega$.
\end{abstract}

\section{Introduction}

In this paper we prove a symmetry result for the heat equation in a certain class of bounded domains $\Omega \subset \mathbb{R}^{n+1}$ and in order to avoid minor notational technicalities we will in the following assume $n \geq 2$. To properly formulate our problem we define for $(X, t)=\left(x_{0}, x_{1}, \ldots, x_{n-1}, t\right) \in \mathbb{R}^{n+1}$ the heat kernel

$$
W(X, t)=(4 \pi t)^{-n / 2} \exp \left(-\frac{|X|^{2}}{4 t}\right) .
$$

For $t \leq 0$ we let $W(X, t) \equiv 0$. For $\lambda>0$ we define

$$
L(\lambda)=\{(X, t): W(X, t)=\lambda\}, D(\lambda)=\{(X, t): W(X, t)>\lambda\} .
$$

2000 Mathematics Subject Classification: 35K05.

Keywords: Heat equation, caloric measure, Green's function, symmetry theorem, free boundary. 
Furthermore for $t>0$

$$
L_{t}(\lambda)=L(\lambda) \cap\{(X, \tau): \tau=t\}, \quad D_{t}(\lambda)=D(\lambda) \cap\{(X, \tau): \tau=t\} .
$$

By $\sigma_{\lambda, t}(\cdot)$ we denote the $n-1$ dimensional Hausdorff measure on $L_{t}(\lambda)$ and $d \sigma_{\lambda}(X, t):=d \sigma_{\lambda, t}(X) d t$ is defined to be the surface measure on $L(\lambda)$. Note that if $(X, t) \in L(\lambda)$ then

$$
\left|\nabla_{X} W(X, t)\right|=\lambda \frac{|X|}{2 t}
$$

and $W(X, t)-\lambda$ can be seen as the Green function of $D(\lambda)$ with pole at $(0,0)$. Furthermore, the associated adjoint caloric measure, $\hat{\omega}$, (defined with respect to $(0,0))$ is absolutely continuous with respect to the surface measure $\sigma_{\lambda}$ and its Radon-Nikodym derivative equals $\lambda|X| /(2 t)$.

For $(X, t) \in \mathbb{R}^{n+1}$ and $r>0$ we set

$$
C_{r}(X, t)=\left\{(Y, s) \in \mathbb{R}^{n+1}:\left|y_{i}-x_{i}\right|<r,|t-s|<r^{2}\right\} .
$$

The geometrical set-up of this paper is what we call local Lip(1,1/2) domains.

Definition 1 Let $\Omega \subset \mathbb{R}^{n+1}$ be a bounded domain and define, for $T>0$, $\Omega_{T}=\Omega \cap\left[\mathbb{R}^{n} \times(0, T)\right]$. Assume that $(0,0) \in \partial_{p} \Omega_{T}$. $\Omega_{T}$ is said to be a local $\operatorname{Lip}(1,1 / 2)$ domain if for every $\left(X_{0}, t_{0}\right) \in \partial \Omega \cap\left[\mathbb{R}^{n} \times(0, T)\right]$ and for a scale $2 \rho=t_{0}^{1 / 2}$ there exists a function $A(x, t)$ and a constant $\delta_{0}=\delta_{0}\left(t_{0}\right)$ such that

$$
|A(x, t)-A(y, s)| \leq \delta_{0}\left(|x-y|+|t-s|^{1 / 2}\right) \quad \text { for } \quad x, y \in \mathbb{R}^{n-1}, \quad t, s \in \mathbb{R}
$$

and such that after a possible rotation in the space variables,

$$
\begin{aligned}
\Omega \cap C_{\rho}\left(X_{0}, t_{0}\right) & =\left\{\left(x_{0}, x, t\right) \in \mathbb{R} \times \mathbb{R}^{n-1} \times \mathbb{R}: x_{0}>A(x, t)\right\} \cap C_{\rho}\left(X_{0}, t_{0}\right), \\
\partial \Omega \cap C_{\rho}\left(X_{0}, t_{0}\right) & =\left\{\left(x_{0}, x, t\right) \in \mathbb{R} \times \mathbb{R}^{n-1} \times \mathbb{R}: x_{0}=A(x, t)\right\} \cap C_{\rho}\left(X_{0}, t_{0}\right) .
\end{aligned}
$$

To properly understand the reason for introducing this notion of local $\operatorname{Lip}(1,1 / 2)$ domains note that the level lines of the heat kernel, i.e., the sets $L(\lambda)$ for $\lambda>0$, define local $\operatorname{Lip}(1,1 / 2)$ domains. In fact if $(X, t) \in L(\lambda)$, $t>0$ close to zero, then

$$
|X|^{2}=-C_{1}(n, \lambda) t-C_{2}(n) t \ln t
$$

for appropriate constants $C_{1}$ and $C_{2}$. This relation shows that $L(\lambda)$ is a local $\operatorname{Lip}(1,1 / 2)$ domain in the sense of Definition 1 with $\operatorname{Lip}(1,1 / 2)$ constants blowing-up as we approach the origin, $(0,0)$. 
Let in the following $\Omega_{T}=\Omega \cap\left[\mathbb{R}^{n} \times(0, T)\right]$ be a local $\operatorname{Lip}(1,1 / 2)$ domain in the sense of Definition 1 and let $\partial_{p} \Omega_{T}$ denote the parabolic boundary of $\Omega_{T}$. Given $(Y, s) \in \Omega_{T}$ we let $G(\cdot, \cdot, Y, s)$ denote Green's function for the heat equation in $\Omega_{T}$ with pole at $(Y, s)$. That is

$\frac{\partial}{\partial t} G(X, t, Y, s)-\Delta G(X, t, Y, s)=\delta((X, t)-(Y, s))$ in $\Omega_{T}$ and $G \equiv 0$ on $\partial_{p} \Omega_{T}$.

Here $\delta$ denotes the Dirac delta function and $\Delta$ is the Laplacian in $X$. We note that $G(Y, s, \cdot, \cdot)$ is the Green's function for the adjoint heat equation with pole at $(Y, s) \in \Omega_{T}$, i.e.,

$$
-\frac{\partial}{\partial t} G(Y, s, \cdot, \cdot)-\Delta G(Y, s, \cdot, \cdot)=\delta(\cdot-(Y, s)) .
$$

Let $\omega(Y, s, \cdot), \hat{\omega}(Y, s, \cdot)$ be the corresponding caloric and adjoint caloric measures for the heat - adjoint heat equation in $\Omega$ defined with respect to $(Y, s) \in \Omega$. In particular this means, by the Riesz representation theorem, that if we define $G(\cdot, \cdot, Y, s) \equiv 0$ on $\left[\mathbb{R}^{n} \times[s, T]\right] \backslash \Omega_{T}$, then $u(\cdot, \cdot)=$ $G(\cdot, \cdot, Y, s)$ is, considered as a function in $\mathbb{R}^{n} \times[s, T]$, subcaloric and for all $\phi \in C_{0}^{\infty}\left(\mathbb{R}^{n} \times(s, T)\right)$,

$$
\int\left[\langle\nabla u, \nabla \phi\rangle-u \phi_{t}\right] d X d t=-\int \phi(X, t) d \hat{\omega}(Y, s, X, t) .
$$

If $\Omega_{T}$ is a local $\operatorname{Lip}(1,1 / 2)$ domain in the sense of Definition 1 then $(0,0) \in$ $\partial_{p} \Omega_{T}$ and in the following we will need an extension of the notion of Green's function to allow $(0,0)$ to be the pole. To be able to make sense of such an extension we impose one more restriction on the set of domains we will consider.

Definition 2 Let $\Omega \subset \mathbb{R}^{n+1}$ be a bounded domain, $T>0$, and assume that $\Omega_{T}$ is a local Lip (1,1/2) domain. If there exists $\lambda>0$ such that $D(\lambda) \cap\left[\mathbb{R}^{n} \times\right.$ $(0, T)] \subset \Omega_{T}$ then $\Omega_{T}$ is said to be a local Lip(1,1/2) domain containing a heat ball.

Let $\Omega_{T}$ and $\lambda$ be as in Definition 2 and define $G>0$ to be the positive solution to the heat equation in $\Omega_{T}$ constructed as $G(Y, s)=W(Y, s)+u(Y, s)$, $(Y, s) \in \Omega_{T}$ where $u(Y, s)$ is the bounded solution to the heat equation in $\Omega_{T}$ with boundary values $u(Y, s)=-W(Y, s)$ in the sense of Perron-WienerBrelot. Note that the existence of $u,-\lambda \leq u \leq 0$, follows from Definition 2 and arguments based on the Perron approach. As any point of $\partial_{p} \Omega_{T}$, except for $(0,0) \in \partial_{p} \Omega_{T}$, is regular with respect to the Dirichlet problem for the heat equation it follows that $G$ has continuous boundary value zero on $\partial_{p} \Omega_{T} \backslash\{(0,0)\}$. We note that $G(\cdot, \cdot, Z, \tau)$ converges uniformly to $G$ on compact subsets of $\Omega_{T} \backslash\{(Y, s): s>0\}$ when $(Z, \tau)$ approaches $(0,0)$ in $D(\lambda)$. 
Also $\hat{\omega}(Z, \tau, \cdot)$ converges weakly as a measure to $\hat{\omega}$ and thus the consequence of the Riesz representation theorem stated above remains valid with $\hat{\omega}(Y, s, \cdot)$ and $G(\cdot, \cdot, Y, s)$ replaced by $\hat{\omega}$ and $G$.

Before we state our main theorem note, as a general remark, that if $A$ is a $\operatorname{Lip}(1,1 / 2)$ function and if $\Omega=\left\{\left(x_{0}, x, t\right) \in \mathbb{R}^{n}: x_{0}>A(x, t)\right\}$ then we can define the surface measure $\sigma$ on $\partial \Omega$ as $d \sigma(X, t)=d \sigma_{t}(X) d t$ where $(X, t) \in \partial \Omega$ and $d \sigma_{t}$ is the $n-1$ dimensional Hausdorff measure on the Lipschitz graph $\partial \Omega_{t}=\left\{\left(x_{0}, x\right) \in \mathbb{R}^{n}: x_{0}=A(x, t)\right\}$.

In this paper we prove the following symmetry theorem using this notion of Green's function of $\Omega_{T}$ with pole at $(0,0) \in \partial_{p} \Omega_{T}$.

Theorem 1 Let $\Omega_{T}$ be a local Lip (1,1/2) domain containing a heat ball in the sense of Definition 2. Let $G, \hat{\omega}$ and $\sigma$ be as above and assume that $\hat{\omega}$ is absolutely continuous with respect to $\sigma$. If

$$
\frac{d \hat{\omega}}{d \sigma}(X, t)=\lambda \frac{|X|}{2 t}
$$

for $\operatorname{all}(X, t) \in \partial_{p} \Omega_{T} \backslash\{(X, t): t=0\}$ and for some $\lambda>0$, then $\partial_{p} \Omega_{T} \subseteq L(\lambda)$ and $G=W-\lambda$ in $\Omega_{T}$.

To put the result in Theorem 1 into perspective we recall that Theorem 1 was proven in [13, Theorem 4] under additional regularity assumptions on $\Omega_{T}$. Furthermore, in addition to the above assumptions, in [13] it was assumed that

$$
\lim _{(Y, s) \rightarrow(X, t)}|\nabla G(Y, s)|=\lambda \frac{|X|}{2 t},
$$

radially, for almost every $(X, t) \in \partial_{p} \Omega_{T} \backslash\{(X, t): t=0\}$. As we will see below our assumption on the adjoint caloric measure implies the existence of this radial limit.

To better understand the additional regularity, beyond being $\operatorname{Lip}(1,1 / 2)$, imposed in [13] we recall that through the works in $[11,6,5]$ it has become clear that from the perspective of parabolic singular integrals and caloric measure the parabolic analogue of the notion of Lipschitz domains, explored in elliptic partial differential equations, is graph domains $\tilde{\Omega} \subset \mathbb{R}^{n+1}$ of the form

$$
\tilde{\Omega}=\left\{\left(x_{0}, x, t\right) \in \mathbb{R} \times \mathbb{R}^{n-1} \times \mathbb{R}: x_{0}>A(x, t)\right\}
$$

where $n \geq 2, A(x, t): \mathbb{R}^{n} \rightarrow \mathbb{R}$ is compactly supported and $A(x, t)$ has additional smoothness beyond being just $\operatorname{Lip}(1,1 / 2)$. 
To formulate the additional regularity assumption we need to introduce some more notation. Let $z=(x, t) \in \mathbb{R}^{n-1} \times \mathbb{R}$ and let $\|z\|$ be the unique positive solution $\rho$ of the equation

$$
\frac{t^{2}}{\rho^{4}}+\sum_{i=0}^{n-1} \frac{x_{i}^{2}}{\rho^{2}}=1
$$

Note that $\left\|\left(\delta x, \delta^{2} t\right)\right\|=\delta\|(x, t)\|$ and we will call $\|z\|$ the parabolic norm of $z$. By definition parabolic $B M O$ is the space of locally integrable functions modulo constants satisfying

$$
\|b\|_{*}:=\sup _{B} \frac{1}{|B|} \int_{B}\left|b(z)-m_{B} b\right| d z<\infty .
$$

Here $z=(x, t), B$ denotes the parabolic ball $B=B_{r}\left(z_{0}\right)=\left\{z \in \mathbb{R}^{n-1} \times \mathbb{R}\right.$ : $\left.\left\|z-z_{0}\right\|<r\right\}$ and $m_{B} b$ denotes the average of the function $b$ over the ball $B$. Let $\widehat{f}(\xi, \tau)$ be the Fourier transform of a function $f$ defined on $\mathbb{R}^{n}$, and let $\xi, \tau$ denote the phase variables. For a function $g \in C_{0}^{\infty}(\mathbb{R})$ we introduce the fractional differentiation operator $D_{1 / 2}$ by

$$
\left(\widehat{D_{1 / 2} g}\right)(\tau):=|\tau|^{1 / 2} \hat{g}(\tau) \text {. }
$$

It is well-known that this definition implies

$$
D_{1 / 2} g(s)=c \int_{\mathbb{R}} \frac{g(s)-g(\tau)}{|s-\tau|^{3 / 2}} d \tau,
$$

for an appropriate constant and whenever $s \in \mathbb{R}$. If $h \in C_{0}^{\infty}\left(\mathbb{R}^{n}\right)$ then by $D_{1 / 2}^{t} h: \mathbb{R}^{n} \rightarrow \mathbb{R}$ we will mean $D_{1 / 2} h(x, \cdot)$ defined a.e. for each $x \in \mathbb{R}^{n-1}$. The domains considered in $[11,6]$ can be defined in the following way.

Definition 3 A Time-Varying domain with defining function $A$ and parameters $a_{1}$ and $a_{2}$, denoted $T V\left(A, a_{1}, a_{2}\right)$, is a domain $\tilde{\Omega} \subset \mathbb{R}^{n+1}$ of the form

$$
\tilde{\Omega}=\left\{\left(x_{0}, x, t\right) \in \mathbb{R} \times \mathbb{R}^{n-1} \times \mathbb{R}: x_{0}>A(x, t)\right\}
$$

where $n \geq 2$ and where the function $A(x, t): \mathbb{R}^{n} \rightarrow \mathbb{R}$ is compactly supported and satisfies

$$
\begin{aligned}
& |A(x, t)-A(y, t)| \leq a_{1}|x-y|, x, y \in \mathbb{R}^{n-1}, t \in \mathbb{R}, \\
& D_{1 / 2}^{t} A \in B M O\left(\mathbb{R}^{n}\right), \quad\left\|D_{1 / 2}^{t} A\right\|_{*} \leq a_{2} .
\end{aligned}
$$


One can prove that if $\tilde{\Omega}$ is $T V\left(A, a_{1}, a_{2}\right)$ for some $a_{1}, a_{2}$ then

$$
|A(x, t)-A(y, s)| \leq \delta_{0}\left(|x-y|+|t-s|^{1 / 2}\right) \quad x, y \in \mathbb{R}^{n-1} \quad t, s \in \mathbb{R}
$$

for some $\delta_{0}=\delta_{0}\left(a_{1}, a_{2}\right)$. The connection between the notion of time-varying domains and caloric - adjoint caloric measure is that if $\tilde{\Omega}$ is $T V\left(A, a_{1}, a_{2}\right)$ for some $a_{1}, a_{2}$ then caloric and adjoint caloric measure are each in a $L^{p}$ reverse Hölder class for some $p>1$ (see [11]). Finally we note that examples of [9] and [12] show that caloric and adjoint caloric measure need not be absolutely continuous with respect to the surface measure $\sigma$ in a graph $\operatorname{Lip}(1,1 / 2)$ domain.

After this digression we note that Theorem 1 was proven in [13, Thm. 4] under the assumption that $\Omega_{T}$ is not just a bounded $\operatorname{Lip}(1,1 / 2)$ domain but that locally the domain is in fact $T V\left(A, a_{1}, a_{2}\right)$ for some $a_{1}, a_{2}$. Important use was made of the above $L^{p}$ results for adjoint caloric measure in a timevarying domain. Therefore our main contribution beyond [13] is that we initially make no assumption on $\Omega_{T}$ apart from that it should be locally $\operatorname{Lip}(1,1 / 2)$ and contain a heat ball in the sense of Definition 2. We in fact show that the assumption on $\hat{\omega}$ in Theorem 1 implies that $\Omega$ locally can be described as a time-varying graph domain and that this is enough to use the argument in [13] to conclude Theorem 1.

We also note that concerning the type of symmetry problems considered in this paper much more progress has been made on the corresponding elliptic problems. In particular if we consider a bounded domain $D \subset \mathbb{R}^{n}$ with $0 \in D$ then we can formulate the following theorem due to Lewis and Vogel [15].

Theorem 2 Assume that $D \subset \mathbb{R}^{n}$ is a bounded domain with $0 \in D$. Let $\mu$ be harmonic measure in $D$ defined with respect to 0 and assume

$$
\mu(B(x, r) \cap \partial D) \leq c r^{n-1}
$$

for all $x \in \partial D$ and $r<r_{0}$. If

$$
\mu=a H^{n-1} \text { on } \partial D
$$

then $D$ is a ball with center at 0 .

In the statement of Theorem $2, H^{n-1}$ is the $(n-1)$-dimensional Hausdorff measure. Note that our theorem, i.e., Theorem 1, gives a parabolic version of Theorem 2 under much stronger hypotheses on the domain. Also note that in [14] it was shown by examples that the conditions in the statement of Theorem 2 are essentially minimal for Theorem 2 to hold. To continue in [20, Theorem 2], Serrin proved the following elliptic symmetry result. 
Theorem 3 Suppose that $D \subset \mathbb{R}^{n}$ is a bounded domain with a $C^{2}$ boundary. If there is a positive solution $u \in C^{2}(\bar{D})$ to the elliptic equation

$$
h\left(u,|\nabla u|^{2}\right) \Delta u+k\left(u,|\nabla u|^{2}\right) \sum_{i, j=1}^{n} u_{x_{i}} u_{x_{j}} u_{x_{i} x_{j}}=l\left(u,|\nabla u|^{2}\right)
$$

where $h, k, l$ are continuously differentiable everywhere with respect to their arguments and if $u$ satisfies the boundary conditions

$$
u=0 \text { and } \frac{\partial u}{\partial n}=a=\text { constant on } \partial D
$$

then $D$ is a ball and $u$ is radially symmetric about the center of $D$.

In the statement of this theorem $\frac{\partial}{\partial n}$ denotes the inner normal derivative of $u$ at a point in $\partial D$. We note that through a sequence of papers, see [17] for references, Lewis and Vogel have made considerable progress on the project to obtain the conclusion of Serrin's theorem under minimal regularity assumptions on $\partial D$ and on the boundary values of $|\nabla u|$. In particular Theorem 2 is one theorem proved as part of this project and recently, see $[16,17]$, similar theorems are also proved for non-linear equations of $p$-Laplace type.

Concerning the proof of our result, Theorem 1, the proof combines results and ideas from [8], [10] and [13]. We believe that by connecting these lines of thought we introduce some important new ideas to the field of parabolic free boundary problems and parabolic symmetry theorems. In particular our local $\operatorname{Lip}(1,1 / 2)$ assumption on $\partial \Omega$ is considerably weaker than other papers we have seen in this field (see for example [1] and [19]). Furthermore, the result of Theorem 1 should be considered as a prototype for the more general parabolic symmetry theorems that we plan to prove in the future.

The rest of the paper is organized as follows. In section 2 we start by listing a few basic estimates for solutions to the heat - adjoint heat equation in domains which are locally $\operatorname{Lip}(1,1 / 2)$. We here have to pay some attention to the fact that we want the lemmas to be valid for the Green function with pole at $(0,0)$ as well. If $\Omega_{T}, \hat{\omega}$ and $G$ are as in the statement of Theorem 1, $\left(X_{0}, t_{0}\right) \in \partial \Omega \cap\left[\mathbb{R}^{n} \times(0, T)\right], 2 \rho=t_{0}^{1 / 2}>0$, we then prove that $C^{-1} \delta(Y, s) \leq G(Y, s) \leq C \delta(Y, s)$ for all $(Y, s) \in \Omega \cap C_{3 \rho / 2}\left(X_{0}, t_{0}\right)$ and that this implies that $\left|G_{y_{i} y_{j}}(Y, s)\right|^{2} G(Y, s)$ satisfies a local Carleson measure type condition. Using our Carleson measure condition we prove that locally $\Omega$ is uniformly rectifiable in the sense of [7] and [8]. In the case of graphs this implies that we can control $\left\|D_{1 / 2}^{t} A\right\|_{*}$ and we can therefore prove that locally $\Omega$ can be described as a time-varying graph domain. Some of the ideas behind the proof of these results can also be found in [10] where a different 
but related problem is considered in the case $n=1$. Finally in section 3 we prove Theorem 1 and we prove, by using the results of section 2 , that the assumptions of Theorem 1 imply that the argument presented in [13] can be reused.

\section{Square functions and uniform rectifiability}

In this section we will prove the following theorem.

Theorem 4 Let $\Omega_{T}, G$ and $\hat{\omega}$ be as in the statement of Theorem 1. Then for each $\left(X_{0}, t_{0}\right) \in \partial \Omega \cap\left[\mathbb{R}^{n} \times(0, T)\right]$ there exists a function $A(x, t)$ such that after a possible rotation in the space variables,

$$
\begin{aligned}
\Omega \cap C_{\rho}\left(X_{0}, t_{0}\right) & =\left\{\left(x_{0}, x, t\right) \in \mathbb{R} \times \mathbb{R}^{n-1} \times \mathbb{R}: x_{0}>A(x, t)\right\} \cap C_{\rho}\left(X_{0}, t_{0}\right), \\
\partial \Omega \cap C_{\rho}\left(X_{0}, t_{0}\right) & =\left\{\left(x_{0}, x, t\right) \in \mathbb{R} \times \mathbb{R}^{n-1} \times \mathbb{R}: x_{0}=A(x, t)\right\} \cap C_{\rho}\left(X_{0}, t_{0}\right),
\end{aligned}
$$

where $2 \rho=t_{0}^{1 / 2}$ and $A(x, t)$ fulfills the regularity conditions, for some constants $a_{1}, a_{2}$, stated in Definition 3.

If $\Omega$ is a $\operatorname{Lip}(1,1 / 2)$ domain $(X, t) \in \partial \Omega, r>0$, then in the following we let $\Delta(X, t, r)=C_{r}(X, t) \cap \partial \Omega$ define our surface cubes.

We start this section by stating a few preliminary lemmas. These lemmas are well established results in the setting of $\operatorname{Lip}(1,1 / 2)$ domains for nonnegative solutions to the heat equation and the adjoint heat equation and we refer the reader to [3], [4], [11] and [18]. In [18] relevant estimates are stated and proved in the general setting of second order parabolic equations in divergence form in $\operatorname{Lip}(1,1 / 2)$ domains. Still we have to be careful as we want these lemmas to apply to the Green function $G$ with pole at $(0,0) \in \partial_{p} \Omega_{T}$ in domains, $\Omega_{T}$, which are local $\operatorname{Lip}(1,1 / 2)$ domains containing a heat ball in the sense of Definition 2. The notion of Green function $G$ with pole at the boundary point $(0,0) \in \partial_{p} \Omega_{T}$ was defined in the introduction. As noted in the introduction if $(Y, s) \in \Omega_{T},(X, t) \in \partial_{p} \Omega_{T}, r>0$ and $2 r<t^{1 / 2}$ then

$G(Y, s)=\lim _{m \rightarrow \infty} G\left(Y, s, X_{m}, t_{m}\right), \hat{\omega}(\Delta(X, t, r))=\lim _{m \rightarrow \infty} \hat{\omega}\left(Y_{m}, s_{m}, \Delta(X, t, r)\right)$

for any sequence of points $\left(X_{m}, t_{m}\right) \in D(\lambda)$ such that $\left(X_{m}, t_{m}\right) \rightarrow(0,0)$, i.e., the sequence converges to the pole at $(0,0)$. In this way several of the estimates for the Green function with pole at a point inside $\Omega_{T}$ can be extended to the Green function with pole at $(0,0) \in \partial_{p} \Omega_{T}$. 
Before we continue we need to introduce some more notation. Suppose $\Omega=\left\{\left(x_{0}, x, t\right) \in \mathbb{R} \times \mathbb{R}^{n-1} \times \mathbb{R}: x_{0}>A(x, t)\right\}$ for some $\operatorname{Lip}(1,1 / 2)$ function $A$ having $\operatorname{Lip}(1,1 / 2)$ constant $\delta_{0}$. If $(X, t)=\left(x_{0}, x, t\right) \in \partial \Omega$ we define

$$
\begin{aligned}
& \bar{A}_{r}(X, t)=\left(x_{0}+100 \delta_{0} r, x, t+r^{2}\right), \\
& A_{r}(X, t)=\left(x_{0}+100 \delta_{0} r, x, t\right), \\
& \underline{A}_{r}(X, t)=\left(x_{0}+100 \delta_{0} r, x, t-r^{2}\right) .
\end{aligned}
$$

As $\Omega_{T}$ is a local $\operatorname{Lip}(1,1 / 2)$ we know that locally, after a possible rotation in the space variables, $\Omega_{T}$ can always be described using such a function $A$ and hence the notation introduced can be used in appropriate local coordinates charts. In the following we let

$$
d\left(F_{1}, F_{2}\right)=\inf \left\{|X-Y|+|s-t|^{1 / 2}:(X, t) \in F_{1},(Y, s) \in F_{2}\right\}
$$

denote the parabolic distance between the sets $F_{1}, F_{2}$ and we let $\delta(Y, s)$ denote the parabolic distance from $(Y, s)$ to the boundary of $\partial \Omega$.

Lemma 5 Let $\Omega \subset \mathbb{R}^{n+1}$ be a Lip $(1,1 / 2)$ domain, $\left(X_{0}, t_{0}\right) \in \partial \Omega, r_{0}>0$ and assume $C_{r_{0}}\left(X_{0}, t_{0}\right) \cap \partial \Omega$ is contained in a Lip(1,1/2)-graph with constant $\delta_{0}$. Let $(X, t) \in C_{r_{0} / 2}\left(X_{0}, t_{0}\right) \cap \partial \Omega$ and suppose that $u$ is a non-negative solution to either the heat or the adjoint heat equation in $\Omega \cap C_{r}(X, t), r<r_{0} / 2$, which vanishes continuously on $\partial \Omega \cap C_{2 r}(X, t)$. There exists $\alpha=\alpha\left(\delta_{0}\right), 0<\alpha<\frac{1}{2}$, and $c=c\left(\delta_{0}\right) \geq 1$ such that if $(Y, s) \in \Omega \cap C_{r}(X, t)$ then

$$
u(Y, s) \leq c\left[\frac{d(\{(Y, s)\},\{(X, t)\})}{r}\right]^{\alpha} \sup _{(Z, \tau) \in \Omega \cap C_{r}(X, t)} u(Z, \tau) .
$$

Proof. This result uses, by its very nature, only local information on the solution $u$ and on the domain and follows by a standard iteration argument.

Lemma 6 Let $\Omega \subset \mathbb{R}^{n+1},\left(X_{0}, t_{0}\right), r_{0}>0, C_{r_{0}}\left(X_{0}, t_{0}\right) \cap \partial \Omega$, u, and $(X, t)$ be as in the previous lemma and let $r<r_{0} /\left(200 \delta_{0}\right)$. If $(Y, s) \in \Omega \cap C_{r / 2}(X, t)$, then there exists $c=c\left(\delta_{0}\right)$

$$
u(Y, s) \leq c u\left(\bar{A}_{r}(X, t)\right)
$$

when $u$ is a solution to the heat equation while

$$
u(Y, s) \leq c u\left(\underline{A}_{r}(X, t)\right)
$$

when $u$ is a solution to the adjoint heat equation in $C_{2 r}(X, t) \cap \Omega$. 
Proof. This lemma is what is usually referred to as a Carleson type lemma and again the proof is of local nature and follows by a standard argument based on Lemma 5. We refer the reader to [3], [4], [11] and [18].

Lemma 7 Assume $\Omega_{T}$ to be a local Lip(1,1/2) domain containing a heat ball and assume $\left(X_{0}, t_{0}\right) \in \partial_{p} \Omega_{T}, 2 \rho=t_{0}^{1 / 2}>0$. Let $r<\rho / 2$ and $A \geq 100$. Then there exists a constant $c$ independent of $(X, t)$ and $r$ such that if

$$
(X, t) \in C_{\rho / 2}\left(X_{0}, t_{0}\right) \cap \partial \Omega \quad \text { and if } \quad|X|^{2} \leq A t, \quad t \geq 4 r^{2}
$$

then

$$
c^{-1} r^{n} G\left(\underline{A}_{r}(X, t)\right) \leq \hat{\omega}(\Delta(X, t, r)) \leq c r^{n} G\left(\bar{A}_{r}(X, t)\right) .
$$

Proof. This lemma relates the value of the Green function with pole at $(0,0) \in \partial_{p} \Omega_{T}$ to the associated adjoint caloric measure. As the pole is on $\partial_{p} \Omega_{T}$ this lemma is not completely obvious. Note, using the notation in the statement of the lemma, that if $(Y, s) \in \Omega_{T}$ with $s<t,|Y-X|^{2} \leq A(t-s)$, $t-s \geq 4 r^{2}$, then there exists $c=c\left(A, \delta_{0}\right) \geq 1$ such that

$$
c^{-1} r^{n} G\left(\underline{A}_{r}(X, t), Y, s\right) \leq \hat{\omega}(Y, s, \Delta(X, t, r)) \leq c r^{n} G\left(\bar{A}_{r}(X, t), Y, s\right) .
$$

For the proof of this well-known relation we refer the reader to [3], [4], [11] and $[18]$. Using this inequality and letting $(Y, s) \rightarrow(0,0)$ through a sequence in $D(\lambda)$ we get Lemma 7 .

Lemma 8 Assume $\Omega_{T}$ to be a local Lip(1,1/2) domain containing a heat ball and assume $\left(X_{0}, t_{0}\right) \in \partial_{p} \Omega_{T}, 2 \rho=t_{0}^{1 / 2}>0$. Let $G$ and $\hat{\omega}$ be as in the statement of Theorem 1. Then there exists a constant $c$ such that for all $(Y, s) \in \Omega \cap C_{3 \rho / 2}\left(X_{0}, t_{0}\right)$

$$
c^{-1} \delta(Y, s) \leq G(Y, s) \leq c \delta(Y, s)
$$

Proof. This is a simple consequence of Lemma 6, Lemma 7 and the assumption that

$$
\frac{d \hat{\omega}}{d \sigma}(X, t)=\lambda \frac{|X|}{2 t}
$$

Our next result is crucial to the arguments in this paper. Theorem 9 below is also proved in [10] in one-space dimension and, though we here formulate the theorem under the assumption that $\Omega$ is a local $\operatorname{Lip}(1,1 / 2)$ domain, the reader will notice that the argument in the proof only requires that $\partial \Omega$ separates $\mathbb{R}^{n+1}$ into two unbounded connected components and satisfies a $(M, R)$ Ahlfors condition (see Definition 4 below). 
Theorem 9 Let $\Omega \subset \mathbb{R}^{n+1}$ be a local Lip(1,1/2) domain with constant $\delta_{0}$. Let $\left(X_{0}, t_{0}\right) \in \partial \Omega \cap\left[\mathbb{R}^{n} \times(0, T)\right]$ and let $2 \rho=t_{0}^{1 / 2}>0$. Assume that there exists a non-negative solution $u$ to the heat or the adjoint heat equation in $\Omega$ which vanishes continuously on $\partial \Omega \cap C_{3 \rho / 2}\left(X_{0}, t_{0}\right)$ and a constant $c$ such that for all $(Y, s) \in \Omega \cap C_{3 \rho / 2}\left(X_{0}, t_{0}\right)$

$$
u(Y, s) \leq c \delta(Y, s)
$$

Then there exists a constant $\hat{c}=\hat{c}(c)$ such that for all $(\hat{X}, \hat{t}) \in \partial \Omega \cap C_{\rho}\left(X_{0}, t_{0}\right)$ and $0<r<\rho / 4$

$$
\sum_{i, j=0}^{n-1} \int_{C_{r}(\hat{X}, \hat{t}) \cap \Omega} u_{y_{i} y_{j}}^{2}(Y, s) u(Y, s) d Y d s+2 \int_{C_{r}(\hat{X}, \hat{t}) \cap \Omega} u_{s}^{2}(Y, s) u(Y, s) d Y d s \leq \hat{c} r^{n+1}
$$

Note that the theorem states that locally the measure

$$
\left[u_{y_{i} y_{j}}^{2}(Y, s) u(Y, s)+2 u_{s}^{2}(Y, s) u(Y, s)\right] d Y d s
$$

is a Carleson measure.

Proof. We will only supply the proof in the case $u$ solves the heat equation, the case of a solution to the adjoint equation being treated similarly. To prove the theorem we consider a Whitney decomposition of $\Omega$ adjusted to $C_{5 \rho / 4}\left(X_{0}, t_{0}\right)$. We obtain such a Whitney decomposition of the part of $\Omega$ located in $C_{5 \rho / 4}\left(X_{0}, t_{0}\right)$ from subdividing $C_{5 \rho / 4}\left(X_{0}, t_{0}\right)$ using the bisection method. Hence we let $\left\{C_{i}=C_{r_{i}}\left(Y_{i}, s_{i}\right)\right\}$ be a the set of all such Whitney cubes located in $\Omega \cap C_{5 \rho / 4}\left(X_{0}, t_{0}\right)$ with center $\left(Y_{i}, s_{i}\right)$ and radius $r_{i}$. We choose this sequence such that $C_{i} \cap C_{j}=\emptyset$ if $i \neq j, \gamma_{1} d\left(C_{i}, \partial \Omega\right) \leq r_{i} \leq \gamma_{2} d\left(C_{i}, \partial \Omega\right)$ and $\bigcup_{i} \bar{C}_{i}=\Omega \cap C_{5 \rho / 4}\left(X_{0}, t_{0}\right)$. Here $\gamma_{1}=\gamma_{1}(n), \gamma_{2}=\gamma_{2}(n)$ and $\gamma_{1}<<$ $\gamma_{2}<<1$. Next let $\eta_{i}$ be a partition of unity, i.e., $\sum \eta_{i} \equiv 1$, adapted to $\left\{C_{i}\right\}$ such that the support of $\eta_{i}$ is a subset of $\bigcup\left\{C_{j}: \bar{C}_{j} \cap \bar{C}_{i} \neq \emptyset\right\}$. We also assume that $\eta_{i}$ is infinitely differentiable with $\eta_{i} \geq c^{-1}$ on $C_{i},\left|\left(\eta_{i}\right)_{y_{j}}\right| \leq c r_{i}^{-1}$, $\left|\left(\eta_{i}\right)_{y_{j} y_{k}}\right| \leq c r_{i}^{-2}$ and $\left|\left(\eta_{i}\right)_{s}\right| \leq c r_{i}^{-2}$. Next we fix $\xi<<1$ and define

$$
\Lambda=\left\{j: C_{j} \cap C_{2 r}(\hat{X}, \hat{t}) \neq \emptyset, \quad r_{j} \geq \xi r\right\}
$$

In the following we will use summation convention and we sum the indicies $i$ and $j$ from 0 to $n-1$. We want to estimate

$$
A:=\sum_{k \in \Lambda} \int u\left(u_{y_{i} y_{j}}\right)^{2} \eta_{k} d Y d s, \quad B:=\sum_{k \in \Lambda} \int u u_{s}^{2} \eta_{k} d Y d s
$$


By integration by parts we have

$$
\begin{aligned}
A= & -\sum_{k \in \Lambda} \int u_{y_{i}} u_{y_{j}} u_{y_{i} y_{j}} \eta_{k} d Y d s-\sum_{k \in \Lambda} \int u u_{y_{j}} \Delta u_{y_{j}} \eta_{k} d Y d s \\
& -\sum_{k \in \Lambda} \int u u_{y_{j}} u_{y_{i} y_{j}}\left(\eta_{k}\right)_{y_{j}} d Y d s \\
= & -\frac{1}{2} \sum_{k \in \Lambda} \int \frac{\partial}{\partial y_{j}}|\nabla u|^{2} u_{y_{j}} \eta_{k} d Y d s-\frac{1}{2} \sum_{k \in \Lambda} \int u \frac{\partial}{\partial s}|\nabla u|^{2} \eta_{k} d Y d s \\
& -\sum_{k \in \Lambda} \int u u_{y_{j}} u_{y_{i} y_{j}}\left(\eta_{k}\right)_{y_{j}} d Y d s .
\end{aligned}
$$

Using integration by parts once more we can conclude that,

$$
\begin{aligned}
A= & \frac{1}{2} \sum_{k \in \Lambda} \int|\nabla u|^{2} \Delta u \eta_{k} d Y d s+\frac{1}{2} \sum_{k \in \Lambda} \int|\nabla u|^{2} u_{y_{j}}\left(\eta_{k}\right)_{y_{j}} d Y d s \\
+ & \frac{1}{2} \sum_{k \in \Lambda} \int \Delta u|\nabla u|^{2} \eta_{k} d Y d s+\frac{1}{2} \sum_{k \in \Lambda} \int u|\nabla u|^{2}\left(\eta_{k}\right)_{s} d Y d s \\
& -\sum_{k \in \Lambda} \int u u_{y_{j}} u_{y_{i} y_{j}}\left(\eta_{k}\right)_{y_{j}} d Y d s .
\end{aligned}
$$

Before continuing we similarly manipulate the term $B$. In fact

$$
\begin{aligned}
B= & \sum_{k \in \Lambda} \int u u_{s}^{2} \eta_{k} d Y d s=\sum_{k \in \Lambda} \int u(\Delta u)^{2} \eta_{k} d Y d s \\
= & -\sum_{k \in \Lambda} \int|\nabla u|^{2} \Delta u \eta_{k} d Y d s-\sum_{k \in \Lambda} \int u u_{y_{i}} \Delta u_{y_{i}} \eta_{k} d Y d s \\
& -\sum_{k \in \Lambda} \int u u_{y_{i}} \Delta u\left(\eta_{k}\right)_{y_{i}} d Y d s .
\end{aligned}
$$

By further manipulations

$$
\begin{aligned}
B= & -\sum_{k \in \Lambda} \int|\nabla u|^{2} \Delta u \eta_{k} d Y d s-\frac{1}{2} \sum_{k \in \Lambda} \int u \frac{\partial}{\partial s}|\nabla u|^{2} \eta_{k} d Y d s \\
& -\sum_{k \in \Lambda} \int u u_{y_{i}} \Delta u\left(\eta_{k}\right)_{y_{i}} d Y d s \\
= & -\sum_{k \in \Lambda} \int|\nabla u|^{2} \Delta u \eta_{k} d Y d s+\frac{1}{2} \sum_{k \in \Lambda} \int \Delta u|\nabla u|^{2} \eta_{k} d Y d s \\
& +\frac{1}{2} \sum_{k \in \Lambda} \int u|\nabla u|^{2}\left(\eta_{k}\right)_{s} d Y d s-\sum_{k \in \Lambda} \int u u_{y_{i}} \Delta u\left(\eta_{k}\right)_{y_{i}} d Y d s .
\end{aligned}
$$


We can therefore conclude that

$$
\begin{aligned}
A+2 B= & \frac{1}{2} \sum_{k \in \Lambda} \int|\nabla u|^{2} u_{y_{j}}\left(\eta_{k}\right)_{y_{j}} d Y d s+\frac{3}{2} \sum_{k \in \Lambda} \int u|\nabla u|^{2}\left(\eta_{k}\right)_{s} d Y d s \\
& -\sum_{k \in \Lambda} \int u u_{y_{j}} u_{y_{i} y_{j}}\left(\eta_{k}\right)_{y_{j}} d Y d s-2 \sum_{k \in \Lambda} \int u u_{y_{i}} \Delta u\left(\eta_{k}\right)_{y_{i}} d Y d s \\
=: & I_{1}+I_{2}+I_{3}+I_{4} .
\end{aligned}
$$

We define $\hat{\Lambda}$ to consist of all Whitney cubes $C_{i}$ such that there exist $j \in \Lambda$ and $k \notin \Lambda, \bar{C}_{i} \cap \bar{C}_{j} \neq \emptyset, \bar{C}_{i} \cap \bar{C}_{k} \neq \emptyset$. Note that $u(Y, s) \leq c \delta(Y, s)$ for $(Y, s) \in \Omega \cap C_{3 \rho / 2}\left(X_{0}, t_{0}\right)$ implies a uniform bound on $|\nabla u(Y, s)|$ for $(Y, s) \in \Omega \cap C_{5 \rho / 4}\left(X_{0}, t_{0}\right)$. We can also assume, by interior regularity for the heat equation, that $\left|u_{y_{i} y_{j}}(Y, s)\right| \leq c \delta(Y, s)^{-1}$ for a uniform constant $c$. As $\left\{\eta_{k}\right\}$ constitutes a partition of unity we get, using these estimates that

$$
\left|I_{1}\right|+\left|I_{2}\right|+\left|I_{3}\right|+\left|I_{4}\right| \leq C \sum_{k \in \hat{\Lambda}} r_{k}^{n-1}
$$

Let $\hat{\Lambda}_{1}$ consist of all $i \in \hat{\Lambda}$ for which there exists $k$ with $\bar{C}_{i} \cap \bar{C}_{k} \neq \emptyset$ and $r_{k} \leq \xi r$. By a simple argument,

$$
\sum_{k \in\left(\hat{\Lambda} \backslash \hat{\Lambda}_{1}\right)} r_{k}^{n-1} \leq C r^{n-1}
$$

Let, for an arbitrary Whitney cube $C_{r_{k}}\left(Y_{k}, s_{k}\right),\left(\hat{Y}_{k}, \hat{s}_{k}\right)$ be a point on $\partial \Omega$ which is closest to $\left(Y_{k}, s_{k}\right)$ is the parabolic metric. Then for some $\gamma_{3}=$ $\gamma_{3}(n)>>1$, as $\partial \Omega$ is $\operatorname{Lip}(1,1 / 2)$,

$$
\sum_{k \in \hat{\Lambda}_{1}} r_{k}^{n-1} \leq C \sum_{k \in \hat{\Lambda}_{1}} \sigma\left(C_{\gamma_{3} r_{k}}\left(\hat{Y}_{k}, \hat{s}_{k}\right) \cap \partial \Omega\right) .
$$

As the surface cubes $\left\{C_{\gamma_{3} r_{k}}\left(\hat{Y}_{k}, \hat{s}_{k}\right) \cap \partial \Omega\right\}$ have finite overlap and as in particular each point $(\hat{X}, \hat{t})$ belongs to at most $c=c(n)$ of these cubes we can conclude that the last term in the previous inequality is bounded by

$$
\sigma\left(\partial \Omega \cap C_{\gamma_{3} r}(\hat{X}, \hat{t})\right) \leq \hat{C} r^{n+1} .
$$

Hence

$$
A+2 B=\sum_{k \in \Lambda} \int u\left(u_{y_{i} y_{j}}\right)^{2} \eta_{k}+u u_{s}^{2} \eta_{k} d Y d s \leq C \sum_{k \in \Lambda} r_{k}^{n-1} \leq \hat{C} r^{n+1} .
$$

Recall that $\Lambda=\left\{j: C_{j} \cap C_{2 r}(\hat{X}, \hat{t}) \neq \emptyset, \quad r_{j} \geq \xi r\right\}$. As the constants in the last inequalities are independent of $\xi$ we can let $\xi \rightarrow 0$ in order to complete the proof. 
Next we show that Theorem 9 implies parabolic uniform rectifiability in the sense of $[7,8]$. In [8] the parabolic Poisson kernel was analyzed in domains not locally given by graphs and instead the geometry was controlled by a certain geometric square function, the boundedness of which implied that on every scale the boundary contained 'big pieces of time-varying graphs'. By a time-varying graph, $T V\left(A, a_{1}, a_{2}\right)$, we here mean graph domains having the regularity defined in Definition 3 (see [7]). We show that this square function can be estimated by the Carleson measure in Theorem 9. We begin by listing and discussing some definitions from [7].

A fundamental assumption in [8] is that $\partial \Omega$ separates $\mathbb{R}^{n+1}$ into two unbounded connected components and that $\partial \Omega$ satisfies a $(M, R)$ Ahlfors condition. To make this precise we, for given a Borel set $F \subset \mathbb{R}^{n+1}$ let $\bar{F}$ and $\partial F$ denote the closure and the boundary of $F$, respectively, and put $\sigma(F)=\int_{F} d \sigma_{t} d t$ where $d \sigma_{t}$ is $n-1$ dimensional Hausdorff measure on the time slice $F \cap\left(\mathbb{R}^{n} \times\{t\}\right)$.

Definition 4 Let $\Omega$ be a connected open set in $\mathbb{R}^{n+1}$. We say that $\partial \Omega$ satisfies a $(M, R)$ Ahlfors condition, $M \geq 4$, if for all $(X, t) \in \partial \Omega$ and $0<r \leq R$

$$
\sigma\left(\partial \Omega \cap C_{r}(X, t)\right) \leq M r^{n+1} .
$$

Using the fact that Hausdorff measure does not increase under a projection we deduce that for $0<r \leq R,(X, t) \in \partial \Omega$,

$$
(r / 2)^{n+1} \leq \sigma\left(\partial \Omega \cap C_{r}(X, t)\right) \leq M r^{n+1},
$$

whenever $\partial \Omega$ separates $\mathbb{R}^{n+1}$ into two unbounded connected components and satisfies a $(M, R)$ Ahlfors condition. Recall that

$$
d\left(F_{1}, F_{2}\right)=\inf \left\{|X-Y|+|s-t|^{1 / 2}:(X, t) \in F_{1},(Y, s) \in F_{2}\right\}
$$

denotes the parabolic distance between the sets $F_{1}, F_{2}$ and for $\Omega$ (such that $\partial \Omega$ separates $\mathbb{R}^{n+1}$ and satisfies a $(M, R)$ Ahlfors condition) we set

$$
\gamma(Z, \tau, r)=\inf _{P}\left[r^{-n-3} \int_{\partial \Omega \cap C_{r}(Z, \tau)} d(\{(Y, s)\}, P)^{2} d \sigma(Y, s)\right] .
$$

Here the infimum is taken over all $n$ dimensional planes $P$ containing a line parallel to the $t$ axis. Let

$$
d \nu(Z, \tau, r)=\gamma(Z, \tau, r) d \sigma(Z, \tau) r^{-1} d r
$$

We say that $\nu$ is a Carleson measure on $\left[\partial \Omega \cap C_{R}(Y, s)\right] \times(0, R)$ if there exists $M_{1}<\infty$ such that whenever $(X, t) \in \partial \Omega$ and $C_{\rho}(X, t) \subset C_{R}(Y, s)$, we have

$$
\nu\left(\left[C_{\rho}(X, t) \cap \partial \Omega\right] \times(0, \rho)\right) \leq M_{1} \rho^{n+1} .
$$


The smallest such $M_{1}$ is called the Carleson norm of $\nu$ on $\left[\partial \Omega \cap C_{R}(Y, s)\right] \times$ $(0, R)$ and we write $\|\nu\|_{+}$for the Carleson norm of $\nu$ if the inequality above holds for all $\rho>0$. The following definition can be found in [7] and [8].

Definition $5 \partial \Omega$ is said to be uniformly rectifiable (in the parabolic sense) if $\|\nu\|_{+}<\infty$ and if the Carleson measure condition stated above holds for all $R>0$. If furthermore $\partial \Omega$ separates $\mathbb{R}^{n+1}$ and is uniformly rectifiable, then $\Omega$ is called a parabolic regular domain.

We are now ready to state and prove our next result.

Theorem 10 Let $\Omega \subset \mathbb{R}^{n+1}$ be a local Lip(1,1/2) domain with constant $\delta_{0}$. Let $\left(X_{0}, t_{0}\right) \in \partial \Omega \cap\left[\mathbb{R}^{n} \times(0, T)\right], 2 \rho=t_{0}^{1 / 2}>0$. Assume that there exists a non-negative solution $u$ to the heat or the adjoint heat equation in $\Omega$ which vanishes continuously on $\partial \Omega \cap C_{3 \rho / 2}\left(X_{0}, t_{0}\right)$ and a constant $c$ such that for all $(Y, s) \in \Omega \cap C_{3 \rho / 2}\left(X_{0}, t_{0}\right)$

$$
c^{-1} \delta(Y, s) \leq u(Y, s) \leq c \delta(Y, s) .
$$

Then

$$
\delta:=\sup _{(X, t) \in \partial \Omega \cap C_{\rho}\left(X_{0}, t_{0}\right), 0<r<\rho /\left(2000 \delta_{0}\right)}\|\nu\|(\Delta(X, t, r) \times[0, r])<\infty
$$

and $\delta=\delta\left(\delta_{0}, c\right)$.

Proof. We will only supply the proof in the case $u$ solves the heat equation, the case of a solution to the adjoint equation being treated similarly. Let $\left(X_{0}, t_{0}\right) \in \partial \Omega \cap\left[\mathbb{R}^{n} \times(0, T)\right]$ and let $2 \rho=t_{0}^{1 / 2}>0$. Let furthermore, $(X, t) \in \partial \Omega \cap C_{\rho}\left(X_{0}, t_{0}\right)$ and $R<\rho /\left(2000 \delta_{0}\right)$. Note that we can without loss of generality assume that $\delta_{0}>1$. By definition we need to prove that

$$
\nu(\Delta(X, t, R) \times(0, R)) \leq C R^{n+1}
$$

for a constant $C$ independent of $(X, t)$ and $R$. By assumption we know that the square function estimate of Theorem 9 is valid and hence, as $c^{-1} \delta(Y, s) \leq$ $u(Y, s)$,

$$
\sum_{i, j=0}^{n-1} \int_{C_{r}(\tilde{X}, \tilde{t}) \cap \Omega} u_{y_{i} y_{j}}^{2}(Y, s) \delta(Y, s) d Y d s \leq \hat{c} r^{n+1}
$$

whenever $(\tilde{X}, \tilde{t}) \in \partial \Omega \cap C_{\rho}\left(X_{0}, t_{0}\right)$ and $r<\rho / 4$.

In the following we consider points $(\tilde{X}, \tilde{t}) \in \partial \Omega \cap C_{\rho}\left(X_{0}, t_{0}\right), r<R$ and we define

$$
\Gamma=\left\{A_{r}(\tilde{X}, \tilde{t})+(0,0, \rho): \quad \rho \in\left(-r^{2}, r^{2}\right)\right\} .
$$


I.e., $\Gamma$ is the line in the $t$-direction connecting the points $\bar{A}_{r}(\tilde{X}, \tilde{t})$ and $\underline{A}_{r}(\tilde{X}, \tilde{t})$. By construction this line is contained in $\Omega$ and for all points $(\tilde{Z}, \tilde{\tau}) \in \Gamma, \delta(\tilde{Z}, \tilde{\tau}) \approx r$. Let $(\hat{X}, \tilde{t})=A_{r}(\tilde{X}, \tilde{t})$ and let $I$ denote the interval $\left(\tilde{t}-r^{2}, \tilde{t}+r^{2}\right)$.

Let $(Y, s) \in \bar{\Omega}$ with $s \in I$ and recall that if $(\tilde{X}, \tilde{t})=\left(\tilde{x}_{0}, \tilde{x}, \tilde{t}\right)$ then by definition $(\hat{X}, \tilde{t})=A_{r}(\tilde{X}, \tilde{t})=\left(\tilde{x}_{0}+100 \delta_{0} r, \tilde{x}, \tilde{t}\right)$. Let $\epsilon \in\left(0,100 \delta_{0}\right)$. Using the mean value theorem we can conclude that there exists $\beta \in\left(\epsilon, 100 \delta_{0}\right)$ such that

$$
u(\hat{X}, \tilde{t})-u\left(\tilde{x}_{0}+\epsilon r, \tilde{x}, \tilde{t}\right)=\left\langle\nabla u\left(\tilde{x}_{0}+\beta r, \tilde{x}, \tilde{t}\right), e_{0}\right\rangle\left(100 \delta_{0}-\epsilon\right) r
$$

where $e_{0}$ is the unit vector in the $x_{0}$-direction. Using Lemma 5 , Lemma 6 and the assumption that $u$ behaves like the parabolic distance to the boundary we also have that there exists a constant $C=C\left(\delta_{0}\right)$ and $\alpha=\alpha\left(\delta_{0}\right)$ such that if $\epsilon<<100 \delta_{0}$ then

$$
u\left(\tilde{x}_{0}+\epsilon r, \tilde{x}, \tilde{t}\right) \leq C \epsilon^{\alpha} \delta(\hat{X}, \tilde{t}) .
$$

Combining we can conclude that

$$
\begin{aligned}
\left\langle\nabla u\left(\tilde{x}_{0}+\beta r, \tilde{x}, \tilde{t}\right), e_{0}\right\rangle\left(100 \delta_{0}-\epsilon\right) r & \geq C_{1}\left(1-C_{2} \epsilon^{\alpha}\right) \delta(\hat{X}, \tilde{t}) \\
& \sim \tilde{C}_{1}\left(1-C_{2} \epsilon^{\alpha}\right) r
\end{aligned}
$$

where the constants $C_{1}, \tilde{C}_{1}$ and $\tilde{C}_{2}$ have the same dependence as the constant $C$. If we let $\left(X^{*}, \tilde{t}\right):=\left(\tilde{x}_{0}+\beta r, \tilde{x}, \tilde{t}\right)$ we have therefore proved that $\left|\nabla u\left(X^{*}, \tilde{t}\right)\right| \geq \tilde{C}$ for some universal constant $\tilde{C}$ and for a point $\left(X^{*}, \tilde{t}\right)$ such that $\delta\left(X^{*}, \tilde{t}\right) \sim r$. To continue we define, for $Y \in \mathbb{R}^{n}$,

$$
A\left(X^{*}, Y, \tilde{t}\right)=u\left(X^{*}, \tilde{t}\right)+\left\langle\nabla u\left(X^{*}, \tilde{t}\right), Y-X^{*}\right\rangle
$$

and we let $P$ denote the hyperplane $\left\{(Z, \tau): A\left(X^{*}, Z, \tilde{t}\right)=0\right\}$. Note that we can conclude, by construction, that $A\left(X^{*}, Y, \tilde{t}\right) \approx d(\{(Y, s)\}, P)$ for all $(Y, s) \in \Delta(\tilde{X}, \tilde{t}, r)$. Using this we can start to estimate the Carleson measure. By definition

$$
\nu(\Delta(X, t, R) \times(0, R)) \leq \int_{0}^{R} \int_{\Delta(X, t, R)} \xi(\tilde{X}, \tilde{t}, r) d \sigma(\tilde{X}, \tilde{t}) d r
$$

where

$$
\xi(\tilde{X}, \tilde{t}, r)=r^{-n-4} \int_{\Delta(\tilde{X}, \tilde{t}, r)} d(\{(Y, s)\}, P)^{2} d \sigma(Y, s)
$$


and where $P$ is the plane constructed. Using the deduction above

$$
\begin{aligned}
\xi(\tilde{X}, \tilde{t}, r) & =r^{-n-4} \int_{\Delta(\tilde{X}, \tilde{t}, r)} d(\{(Y, s)\}, P)^{2} d \sigma(Y, s) \\
& \approx r^{-n-4} \int_{\Delta(\tilde{X}, \tilde{t}, r)} A\left(X^{*}, Y, \tilde{t}\right)^{2} d \sigma(Y, s) \\
& \leq 2 r^{-n-4} \int_{\Delta(\tilde{X}, \tilde{t}, r)} A(\hat{X}, Y, \tilde{t})^{2} d \sigma(Y, s) \\
& +2 r^{-n-4} \int_{\Delta(\tilde{X}, \tilde{t}, r)}\left|A\left(X^{*}, Y, \tilde{t}\right)-A(\hat{X}, Y, \tilde{t})\right|^{2} d \sigma(Y, s) .
\end{aligned}
$$

In fact we decompose the term on the second line in the last display one step further and get

$$
\begin{aligned}
\xi(\tilde{X}, \tilde{t}, r) & \leq 2 r^{-n-4} \int_{\Delta(\tilde{X}, \tilde{t}, r)} A(\hat{X}, Y, s)^{2} d \sigma(Y, s) \\
& +2 r^{-n-4} \int_{\Delta(\tilde{X}, \tilde{t}, r)}|A(\hat{X}, Y, \tilde{t})-A(\hat{X}, Y, s)|^{2} d \sigma(Y, s) \\
& +2 r^{-n-4} \int_{\Delta(\tilde{X}, \tilde{t}, r)}\left|A\left(X^{*}, Y, \tilde{t}\right)-A(\hat{X}, Y, \tilde{t})\right|^{2} d \sigma(Y, s) .
\end{aligned}
$$

Let $(Y, s) \in \Delta(\tilde{X}, \tilde{t}, r)$ with $s \in I$. Using Taylor's formula,

$$
u(Y, s)=u(\hat{X}, s)+\langle\nabla u(\hat{X}, s), Y-\hat{X}\rangle+\int_{\hat{X}}^{Y}\left\langle D^{2} u(Z, s), Z-Y\right\rangle d l .
$$

Here the last integral is interpreted as the second directional derivative of $u$ taken along the line segment $l$ from $(\hat{X}, s)$ to $(Y, s)$ (with $Z$ on $l$ ). Using the fact that $u=0$ on $\partial \Omega$, Taylor's formula as above and Schwarz's inequality we get for all $s \in I$,

$$
|A(\hat{X}, Y, s)|^{2} \leq c r \int_{\hat{X}}^{Y} \delta(Z, s)^{2} \sum_{i, j=0}^{n-1} u_{z_{i} z_{j}}^{2}(Z, s) d l .
$$

Furthermore, using the mean value theorem in calculus and interior estimates for derivatives of second order of solutions to the heat equation we can conclude that if $(Y, s) \in \Delta(\tilde{X}, \tilde{t}, r)$, then

$$
|A(\hat{X}, Y, \tilde{t})-A(\hat{X}, Y, s)|^{2} \leq c r^{-n} \int_{C_{2 r}(\hat{X}, \tilde{t})} \delta(Z, s)^{2} \sum_{i, j=0}^{n-1} u_{z_{i} z_{j}}^{2}(Z, s) d Z d s
$$


Left is to estimate $\left|A\left(X^{*}, Y, \tilde{t}\right)-A(\hat{X}, Y, \tilde{t})\right|$. But again using Taylor's formula, Schwarz's inequality and replacing pointwise estimates by averages we get, if $(Y, s) \in \Delta(\tilde{X}, \tilde{t}, r)$,

$$
\begin{aligned}
\left|A\left(X^{*}, Y, \tilde{t}\right)-A(\hat{X}, Y, \tilde{t})\right|^{2} & \leq c r \int_{X^{*}}^{\hat{X}} \delta(Z, \tilde{t})^{2} \sum_{i, j=0}^{n-1} u_{z_{i} z_{j}}^{2}(Z, \tilde{t}) d l^{*} \\
& \leq c r^{-n} \int_{C_{110 \delta_{0} r}(\tilde{X}, \tilde{t})} \delta(Z, s)^{2} \sum_{i, j=0}^{n-1} u_{z_{i} z_{j}}^{2}(Z, s) d Z d s
\end{aligned}
$$

where $l^{*}$ is the line from $\left(X^{*}, \tilde{t}\right)$ to $(\hat{X}, \tilde{t})$. Continuing our previous deduction, using simple arguments, we find that

$$
\xi(\tilde{X}, \tilde{t}, r) \leq c r^{-n-3} \int_{C_{110 \delta_{0} r}(\tilde{X}, \tilde{t})} \delta(Z, s)^{2} \sum_{i, j=0}^{n-1} u_{z_{i} z_{j}}^{2}(Z, s) d Z d s .
$$

Hence

$$
\nu(\Delta(X, t, R) \times(0, R)) \leq \int_{0}^{R} \int_{\Delta(X, t, R)} \xi(\tilde{X}, \tilde{t}, r) d \sigma(\tilde{X}, \tilde{t}) d r
$$

Using the expression for $\xi(\tilde{X}, \tilde{t}, r)$ we obtain, after interchanging the order of integration, that the right-hand side in the last expression is dominated, for some small $\gamma>0$, by

$$
\begin{aligned}
& c \int_{C_{200 \delta_{0} R}(X, t)} \delta(Z, s)^{2} \sum_{i, j=0}^{n-1} u_{z_{i} z_{j}}^{2}(Z, s)\left[\int_{\gamma \delta(Z, s)}^{\infty} r^{-2} d r\right] d Z d s \\
= & c \int_{C_{200 \delta_{0} R}(X, t)} \delta(Z, s) \sum_{i, j=0}^{n-1} u_{z_{i} z_{j}}^{2}(Z, s) d Z d s \leq c R^{n+1} .
\end{aligned}
$$

This completes the proof.

Consider a parabolic cube $C_{\rho}(X, t) \subset \mathbb{R}^{n+1}$ for some $(X, t) \in \mathbb{R}^{n+1}$. In the following we let $\hat{C}_{\rho}(X, t)$ denote the projection of $C_{\rho}(X, t)$ onto $\mathbb{R}^{n-1} \times \mathbb{R}$ and if $\Omega_{\psi}=\left\{\left(x_{0}, x, t\right) \in \mathbb{R} \times \mathbb{R}^{n-1} \times \mathbb{R}: x_{0}>\psi(x, t)\right\}, \psi$ is assumed to be $\operatorname{Lip}(1,1 / 2)$, then we let $\gamma_{\psi}=\gamma, \nu_{\psi}=\nu$ be defined, see the definitions above Definition 5, with respect to the graph $\left\{(\psi(x, t), x, t):(x, t) \in \mathbb{R}^{n}\right\}$.

In order to prove Theorem 4 we will also need the following theorem, Theorem 11, and we note that Theorem 4 follows by combining Lemma 8, Theorem 10 and Theorem 11 in a standard argument based on a partition of unity. 
Theorem 11 Let $\Omega \subset \mathbb{R}^{n+1}$ be a local Lip(1,1/2) domain with constant $\delta_{0}$, $\left(X_{0}, t_{0}\right) \in \partial \Omega \cap\left[\mathbb{R}^{n} \times(0, T)\right], \hat{\rho}>0$ and assume that $C_{\rho}\left(X_{0}, t_{0}\right) \subset\left[\mathbb{R}^{n} \times(0, T)\right]$, $\rho=100 \hat{\rho}$, and

$$
\begin{aligned}
\Omega \cap C_{\rho}\left(X_{0}, t_{0}\right) & =\left\{\left(x_{0}, x, t\right) \in \mathbb{R} \times \mathbb{R}^{n-1} \times \mathbb{R}: x_{0}>A(x, t)\right\} \cap C_{\rho}\left(X_{0}, t_{0}\right), \\
\partial \Omega \cap C_{\rho}\left(X_{0}, t_{0}\right) & =\left\{\left(x_{0}, x, t\right) \in \mathbb{R} \times \mathbb{R}^{n-1} \times \mathbb{R}: x_{0}=A(x, t)\right\} \cap C_{\rho}\left(X_{0}, t_{0}\right) .
\end{aligned}
$$

Define

$$
\psi(x, t)=\phi(x, t) A(x, t), \quad(x, t) \in \mathbb{R}^{n-1} \times \mathbb{R},
$$

where $\phi \in C_{0}^{\infty}\left(\hat{C}_{2 \hat{\rho}}\left(X_{0}, t_{0}\right)\right), \phi \equiv 1$ on $\hat{C}_{3 \hat{\rho} / 2}\left(X_{0}, t_{0}\right)$. Let

$$
\Omega_{\psi}=\left\{\left(x_{0}, x, t\right) \in \mathbb{R} \times \mathbb{R}^{n-1} \times \mathbb{R}: x_{0}>\psi(x, t)\right\} .
$$

If

$$
\delta:=\sup _{(X, t) \in \partial \Omega \cap C_{10 \hat{\rho}}\left(X_{0}, t_{0}\right), 0<r<10 \hat{\rho}}\left\|\nu_{A}\right\|(\Delta(X, t, r) \times[0, r])<\infty
$$

for some $\delta>0$, then

$$
\delta_{\psi}:=\sup _{(X, t) \in \partial \Omega_{\psi}, r>0}\left\|\nu_{\psi}\right\|(\Delta(X, t, r) \times[0, r])<\infty
$$

and $\left\|D_{1 / 2}^{t} \psi\right\|_{*}<\infty$.

Proof. That $\delta_{\psi}<\infty$ implies $\left\|D_{1 / 2}^{t} \psi\right\|_{*}<\infty$ is proved in [8, p. 287-292] and hence we only need to prove that if $\delta<\infty$ then $\delta_{\psi}<\infty$. In fact the latter statement is more or less trivial but we include a proof for the convenience of the reader. We furthermore note that in the following we do not have to keep track of the dependence of the constants appearing in our deductions, explicitly and at each instance, as we only want to prove finiteness of $\delta_{\psi}$. Therefore, in the following $C>0$ will be a generic constant depending on $n$, $\delta_{0}, \hat{\rho}$ and the supremum of $|A|,\left|\phi_{x_{i}}\right|,\left|\phi_{x_{i} x_{j}}\right|$ and $\left|\phi_{t}\right|$ over the set $\hat{C}_{2 \hat{\rho}}\left(X_{0}, t_{0}\right)$.

Defining, for $r>0,(\hat{x}, \hat{t}) \in \mathbb{R}^{n}$, with $C_{r}(\hat{x}, \hat{t})=\left\{(y, s) \in \mathbb{R}^{n}:\left|y_{i}-\hat{x}_{i}\right|<\right.$ $\left.r,|\hat{t}-s|<r^{2}\right\}$

$$
\kappa_{\psi}(\hat{x}, \hat{t}, r)=r^{-(n+3)} \inf _{L} \int_{C_{r}(\hat{x}, \hat{t})}|\psi(y, s)-L(y)|^{2} d y d s
$$

where the infimum is over all linear functions of $y$ (only) we can conclude, by arguing as in $[8$, formula (2.3)-(2.5)], that

$$
C^{-1} \kappa_{\psi}(\hat{x}, \hat{t}, r) \leq \gamma_{\psi}(\hat{X}, \hat{t}, r) \leq C \kappa_{\psi}(\hat{x}, \hat{t}, r)
$$

for $r>0,(\hat{x}, \hat{t}) \in \mathbb{R}^{n}$ and for a constant $C>0$. Here $(\hat{X}, \hat{t})=\left(\hat{x}_{0}, \hat{x}, \hat{t}\right)$. Note that $\kappa_{\psi}(\hat{x}, \hat{t}, r)=0$ for all $r>0$ and $(\hat{x}, \hat{t})$ such that $\hat{C}_{2 \hat{\rho}}\left(X_{0}, t_{0}\right) \cap C_{r}(\hat{x}, \hat{t})=\emptyset$. 
Hence we can assume that $\hat{C}_{2 \hat{\rho}}\left(X_{0}, t_{0}\right) \cap C_{r}(\hat{x}, \hat{t}) \neq \emptyset$. We now consider a number of cases. Firstly we assume that $(\hat{x}, \hat{t}) \in \hat{C}_{4 \hat{\rho}}\left(X_{0}, t_{0}\right)$ and consider the cases $r \leq 2 \hat{\rho}$ and $r>2 \hat{\rho}$. We claim that in case $r \leq 2 \hat{\rho}$ then

$$
\kappa_{\psi}(\hat{x}, \hat{t}, r) \leq C\left[r^{2}+\kappa_{A}(\hat{x}, \hat{t}, r)\right] .
$$

To prove this claim we let $L_{1}(y)=\phi(\hat{x}, \hat{t})+L_{2}(y), L_{2}(y)=\left\langle\nabla_{z} \phi(\hat{x}, \hat{t}),(y-\hat{x})\right\rangle$ and decompose $\phi(y, s) A(y, s)=\phi_{1}(y, s)+\phi_{2}(y, s)+\phi_{3}(y, s)$ where

$$
\begin{aligned}
\phi_{1}(y, s) & =\left(\phi(y, s)-L_{1}(y)\right) A(y, s), \\
\phi_{2}(y, s) & =\left\langle\nabla_{z} \phi(\hat{x}, \hat{t}),(y-\hat{x})\right\rangle(A(y, s)-A(\hat{x}, \hat{t})), \\
\phi_{3}(y, s) & =A(\hat{x}, \hat{t}) L_{2}(y)+\phi(\hat{x}, \hat{t}) A(y, s) .
\end{aligned}
$$

If $L(y)$ is an arbitrary linear functions of $y$ then

$$
\begin{aligned}
& \left|\phi(y, s) A(y, s)-\phi(\hat{x}, \hat{t}) L(y)-A(\hat{x}, \hat{t}) L_{2}(y)\right|^{2} \\
& \quad \leq C\left[\left|\phi_{1}(y, s)\right|^{2}+\left|\phi_{2}(y, s)\right|^{2}+|\phi(\hat{x}, \hat{t})|^{2}|A(y, s)-L(y)|^{2}\right] .
\end{aligned}
$$

Hence, using that $\phi$ is infinitely differentiable, the anisotropic scaling of the parabolic cube $C_{r}(\hat{x}, \hat{t})$, Taylor's formula and the $\operatorname{Lip}(1,1 / 2)$ property of $A$ we can conclude that the claim is true. Therefore,

$$
\kappa_{\psi}(\hat{x}, \hat{t}, r) \leq C\left[r^{2}+k_{A}(\hat{x}, \hat{t}, r)\right] \leq C\left[r^{2}+\gamma_{A}(\hat{X}, \hat{t}, r)\right] .
$$

Furthermore, in case $r>2 \hat{\rho}$ we get, using Taylor's formula, for $\phi$, with respect to a point outside of the support of $\phi$, but located at most $4 \hat{\rho}$ away from any point in the set $C_{r}(\hat{x}, \hat{t})$, and taking $L \equiv 0$,

$$
\kappa_{\psi}(\hat{x}, \hat{t}, r) \leq r^{-(n+3)} \int_{C_{r}(\hat{x}, \hat{t})}|\psi(y, s)|^{2} d y d s \leq C\left(\frac{\hat{\rho}}{r}\right)^{n+3}
$$

Finally we note it follows, by reusing the last argument, that the estimate

$$
\kappa_{\psi}(\hat{x}, \hat{t}, r) \leq C(\hat{\rho} / r)^{n+3}
$$

is also true in the case $(\hat{x}, \hat{t}) \notin \hat{C}_{4 \hat{\rho}}\left(X_{0}, t_{0}\right)$ and $r>2 \hat{\rho}$.

To complete the proof we let $(z, \tau) \in \mathbb{R}^{n}$ and $\tilde{\rho}>0$. Splitting the domain of integration, based on the analysis above, we see that

$$
\int_{0}^{\tilde{\rho}} \int_{C_{\tilde{\rho}}(z, \tau)} \lambda^{-1} \kappa_{\psi}(\hat{x}, \hat{t}, \lambda) d \hat{x} d \hat{t} d \lambda \leq C[1+\delta] \tilde{\rho}^{n+1}
$$

where $\delta$ is the the localized Carleson norm of $\nu_{A}$ as defined in the statement of the theorem. Hence we can conclude that $\delta_{\psi}<\infty$ and the proof is complete. 


\section{Proof of Theorem 1}

In this section we will prove Theorem 1. First from the assumptions in Theorem 1 it follows that Theorem 4 is valid, i.e., for each $\left(X_{0}, t_{0}\right) \in \partial \Omega \cap$ $\left[\mathbb{R}^{n} \times(0, T)\right]$ there exists a function $A(x, t)$ such that after a possible rotation in the space variables,

$$
\begin{aligned}
\Omega \cap C_{\rho}\left(X_{0}, t_{0}\right) & =\left\{\left(x_{0}, x, t\right) \in \mathbb{R} \times \mathbb{R}^{n-1} \times \mathbb{R}: x_{0}>A(x, t)\right\} \cap C_{\rho}\left(X_{0}, t_{0}\right), \\
\partial \Omega \cap C_{\rho}\left(X_{0}, t_{0}\right) & =\left\{\left(x_{0}, x, t\right) \in \mathbb{R} \times \mathbb{R}^{n-1} \times \mathbb{R}: x_{0}=A(x, t)\right\} \cap C_{\rho}\left(X_{0}, t_{0}\right),
\end{aligned}
$$

where $2 \rho=t_{0}^{1 / 2}$ and $A(x, t)$ fulfills the regularity conditions, for some constants $a_{1}, a_{2}$, stated in Definition 3. We can therefore use the results of [11] to conclude that

$$
\lim _{(Y, s) \rightarrow(X, t)}\left|\nabla_{Y} G(Y, s)\right|=\frac{d \hat{\omega}}{d \sigma}(X, t)=\lambda \frac{|X|}{2 t}
$$

non-tangentially for almost every $(X, t) \in \partial \Omega \cap\left[\mathbb{R}^{n} \times(0, T)\right]$.

Again using the results of [11] on the $L^{p}$-solvability of the Dirichlet problem for some $p>1$ one can show that each component of $\nabla G$ can be realized as a Poisson integral and it follows by an appropriate approximation argument (see [13, p. 359-360]) that if $t_{1}$ and $t_{2}$ are such that $0<t_{1}<t_{2}<T$, then

$$
\frac{\lambda}{2} \int_{t_{1}}^{t_{2}}\left(\int_{\partial \Omega_{t}}|X| d \sigma_{t}(X)\right) \frac{d t}{t}=\int_{\Omega_{t_{1}}} G\left(Y, t_{1}\right) d Y-\int_{\Omega_{t_{2}}} G\left(Y, t_{2}\right) d Y .
$$

This equality is simply a consequence of our knowledge of the boundary values of $|\nabla G(Y, s)|$ and partial integration. Note that

$$
\int_{\Omega_{t_{1}}} G\left(Y, t_{1}\right) d Y \leq \int_{\mathbb{R}^{n}} W\left(Y, t_{1}\right) d Y=1 .
$$

If we let $t_{1} \rightarrow 0$ in the identity above we can therefore conclude that

$$
\frac{\lambda}{2} \int_{0}^{t_{2}}\left(\int_{\partial \Omega_{t}}|X| d \sigma_{t}(X)\right) \frac{d t}{t}+\int_{\Omega_{t_{2}}} G\left(Y, t_{2}\right) d Y \leq 1
$$

for all $t_{2} \in(0, T]$. Recall that for $\lambda>0$

$$
L(\lambda)=\{(X, t): W(X, t)=\lambda\}, D(\lambda)=\{(X, t): W(X, t)>\lambda\} .
$$

Furthermore for $t>0$

$$
L_{t}(\lambda)=L(\lambda) \cap\{(X, \tau): \tau=t\}, \quad D_{t}(\lambda)=D(\lambda) \cap\{(X, \tau): \tau=t\} .
$$


Arguing as in [13, p.360-361] we can conclude that there exists $\lambda_{0}$ and $t_{2}$ such that $\lambda \geq \lambda_{0}, G \geq W-\lambda_{0}$ in $\Omega \cap\left[\mathbb{R}^{n} \times\left(0, t_{2}\right)\right]$ and, for all $t \in\left(0, t_{2}\right)$,

$$
\min _{X \in \partial \Omega_{t}}|X| \geq \max _{X \in L_{t}\left(\lambda_{0}\right)}|X|
$$

Using this and the inequality above we can therefore conclude that

$$
\begin{array}{r}
\frac{\lambda_{0}}{2} \int_{0}^{t_{2}}\left(\int_{L_{t}\left(\lambda_{0}\right)}|X| d \sigma_{\lambda_{0}, t}(X)\right) \frac{d t}{t}+\int_{D_{t_{2}}\left(\lambda_{0}\right)}\left(W\left(Y, t_{2}\right)-\lambda_{0}\right) d Y \\
\leq \frac{\lambda}{2} \int_{0}^{t_{2}}\left(\int_{\partial \Omega_{t}}|X| d \sigma_{t}(X)\right) \frac{d t}{t}+\int_{\Omega_{t_{2}}} G\left(Y, t_{2}\right) d Y \leq 1 .
\end{array}
$$

Here equality only holds if

$$
G \equiv W-\lambda_{0} \quad \text { in } \quad \Omega \cap\left[\mathbb{R}^{n} \times\left(0, t_{2}\right)\right] \quad \text { and } \quad \lambda_{0}=\lambda .
$$

By a simple calculation using the divergence theorem

$$
\frac{\lambda_{0}}{2} \int_{0}^{t_{2}}\left(\int_{L_{t}\left(\lambda_{0}\right)}|X| d \sigma_{\lambda_{0}, t}(X)\right) \frac{d t}{t}+\int_{D_{t_{2}}\left(\lambda_{0}\right)}\left(W\left(Y, t_{2}\right)-\lambda_{0}\right) d Y=1 .
$$

Hence equality must hold and $G \equiv W-\lambda$ in $\Omega \cap\left[\mathbb{R}^{n} \times(0, T)\right]$ and the proof of Theorem 1 is complete.

\section{References}

[1] Athanasopoulos, I., Caffarelli, L. and Salsa, S.: Caloric functions in Lipschitz domains and the regularity of solutions to phase transition problems. Ann. of Math. (2) 143 (1996), no. 3, 413-434.

[2] Doob, J. L.: Classical potential theory and its probablilistic counterpart. Grundlehren der Mathematischen Wissenschaften 262. Springer-Verlag, New York, 1984.

[3] Fabes, E. And SAFonov, M.: Behavior near the boundary of positive solutions of second order parabolic equations. In "Proceedings of the conference dedicated to Professor Miguel de Guzmán (El Escorial, 1996)". J. Fourier Anal. Appl. 3 (1997), Special Issue, 871-882.

[4] Fabes, E., Safonov, M. And Yuan, Y.: Behavior near the boundary of positive solutions of second order parabolic equations, II. Trans. Amer. Math. Soc. 351 (1999), no. 12, 4947-4961.

[5] Hofmann, S.: Parabolic singular integrals of Calderón type, rough operators, and caloric layer potentials. Duke Math. J. 90 (1997), no. 2, 209-259. 
[6] Hofmann, S. And Lewis, J.: $L^{2}$ Solvability and representation by caloric layer potentials in time-varying domains. Ann. of Math. (2) 144 (1996), no. 2, 349-420.

[7] Hofmann, S., Lewis, J. And Nyström, K.: Existence of big pieces of graphs for parabolic problems. Ann. Acad. Sci. Fenn. Math. 28 (2003), no. $2,355-384$.

[8] Hofmann, S., Lewis, J. And Nyström, K.: Caloric measure in parabolic flat domains. Duke Math. J. 122 (2004), no. 2, 281-346.

[9] Kaufmann, R. And Wu, J. M.: Parabolic measure on domains of class $\operatorname{Lip}_{1 / 2}$. Compositio Math. 65 (1988), no. 2, 201-207.

[10] LEWIS, J.: On symmetry and uniform rectifiability arising from some overdetermined elliptic and parabolic boundary conditions. In The p-harmonic equation and recent advances in analysis, 175-187. Contemp. Math. 370. Amer. Math. Soc., Providence, 2005.

[11] Lewis, J. And Murray, M.: The method of layer potentials for the heat equation in time-varying domains. Mem. Amer. Math. Soc. 114 (1995), no. 545 .

[12] Lewis, J. And Silver, J.: Parabolic measure and the Dirichlet problem for the heat equation in two dimensions. Indiana Univ. Math. J. 37 (1988), no. $4,801-839$.

[13] Lewis, J. And Vogel, A.: On some almost everywhere symmetry theorems. In Nonlinear diffusion equations and their equilibrium states, 3 (Gregynog, 1989), 347-374. Progr. Nonlinear Differential Equations Appl. 7. Birkhäuser, Boston, 1992.

[14] Lewis, J. And Vogel, A.: On pseudospheres that are quasispheres. Rev. Mat. Iberoamericana 17 (2001), no. 2, 221-255.

[15] Lewis, J. And Vogel, A.: A symmetry theorem revisited. Proc. Amer. Math. Soc. 130 (2001), no. 2, 443-451.

[16] Lewis, J. And Vogel, A.: Symmetry theorems and uniform rectifiability. Boundary Value Problems special issue: "Harnack Estimates, Positivity and Local Behavior of the Degenerate and Singular Equations" vol. 2007, Article ID 30190, 59 pages, 2007. doi:10.1155/2007/30190.

[17] Lewis, J. And Vogel, A.: Uniqueness in a free boundary problem. Comm. Partial Differential Equations 31 (2006), no. 10-12, 1591-1614.

[18] Nyström, K.: The Dirichlet problem for second order parabolic operators. Indiana Univ. Math. J. 46 (1996), no. 1, 183-245.

[19] Petrosyan, A.: On existence and uniqueness in a free boundary problem from combustion. Comm. Partial Differential Equations 27 (2002), no. 3-4, 763-789.

[20] Serrin, J.: A symmetry problem in potential theory. Arch. Rational Mech. Anal. 43 (1971), 304-318. 
[21] Vogel, A.: Symmetry and regularity for general regions having a solution to certain overdetermined boundary value problems. Atti Sem. Mat. Fis. Univ. Modena 40 (1992), no. 2, 443-484.

Recibido: 21 de junio de 2005.

John L. Lewis Department of Mathematics University of Kentucky Lexington, KY 40506-0027, USA john@ms. uky . edu

Kaj Nyström Department of Mathematics Umeå University S-90187 Umeå, Sweden kaj.nystrom@math. umu.se 